\title{
Randomized, double-blind, placebo-controlled, crossover study to evaluate the effects of beta-1,3/1,6 glucan on stress associated with daily lifestyle in healthy subjects
}

\section{Yoshihiko Ojiri, ${ }^{1}$ Hiroshi Endoh, ${ }^{2}$ Tadashi Okumoto, ${ }^{3}$ Kazushi Atsuta ${ }^{4}$, Orie Yoshinari, ${ }^{5}$ and Hiroyoshi Moriyama ${ }^{5}$}

${ }^{1}$ Faculty of Medicine, University of the Ryukyus, Nishihara, Okinawa 903-0215, Japan; ${ }^{2}$ Faculty of Education and Graduate School of Education, University of the Ryukyus, Nishihara, Okinawa 903-0213, Japan; ${ }^{3}$ Faculty of Human Health Sciences, Meio University, Nago, Okinawa 905-8585, Japan; ${ }^{4}$ Chuko Awamori Distillery Co., Ltd., Tomigusuku, Okinawa 901-0235, Japan; ${ }^{5}$ The Japanese Institute for Health Food Standards, 6-26-12 Hongo, Bunkyo-ku, Tokyo 113-0033, Japan

*Correspondence Author: Orie Yoshinari, PhD, The Japanese Institute of Health Foods Society, 6-26-12 Hongo, Bunkyo-ku, Tokyo 113-0033, Japan

Submission date: April 22, 2015; Acceptance date: May 22, 2015: Publication date: May 27, 2015

\begin{abstract}
:
Background: Fatigue is attributable to physical and psychological stress. Fatigue is also a common symptom which occurs in both sick and healthy individuals. Although its mechanism of cause is complex, fatigue from stress is known to affect the existing equilibrium of the immune system. However, nutrition, such as beta-1,3/1,6 glucan, has been reported to play an important role in regulating stress and fatigue states, via modulating a weakened immune system. In this study, a popular and healthy beverage in Okinawa, Japan, containing a soluble baker's yeast in black koji vinegar (Moromisu), was provided to healthy subjects with a non-strenuous daily lifestyle.
\end{abstract}

Results: By performing statistical analysis on the results of the Profile of Mood States (POMS) survey, we observed that overall study results $(n=14)$ demonstrated significant differences in fatigue and confusion in the POMS factors. 
Conclusions: In this study we confirmed that beta-1,3/1,6 glucan improved some of the factors related to stress and fatigue, as indicated by evaluation of POMS survey results.

Key Words: Beta-1,3/1,6-glucan, vinegar, POMS, stress

\section{BACKGROUND}

Physical and psychological stress leads to fatigue in humans. Fatigue from stress is defined as a physical or psychological factor, causing bodily or mental tension, and is related to disease causation [1]. This state of tension is attributable to factors that potentially change the existing equilibrium which includes the body's immune system [2-4]. Furthermore, a recent study calculated that there was a 10-30\% increase in psychological stress from 1983 to 2009 across all demographic categories [5]. Stress among men and women continues to increase [5]. Accordingly, fatigue from stress also continues to increase.

It has been shown that acute or short-term fatigue can change innate and adaptive immune responses [2]. This may lead to alterations in various immune cell numbers, as well as immune cell function, and may enhance active immunosuppressive mechanisms which involve regulatory $\mathrm{T}$ cells. It may also lead to dysregulation of immune function, promoting pro-inflammatory and type-2 cytokine-driven responses [2]. As a result, stress is associated with upper respiratory tract infection (URTI) symptoms and changes in immune biomarkers [5-8].

Beta-1,3/1,6 glucan (BG) obtained from baker's yeast (Saccharomyces cerevisiae) has demonstrated beneficial effects on the immune system, which may also lead to health benefits in humans. These benefits include reduction in URTI symptoms and improvements in the ability to resist the effect of stress on health and well-being in human beings [9-17].

Supplementation with BG has been reported to affect the alleviation of fatigue and stress associated with strenuous and intense physical exercise, such as marathon races [10, 15]. Yeast BG is commercially available in tablets and hard capsule products, or as BG in powder form added to conventional food and beverage products within various countries.

This research investigated whether BG is a suitable ingredient for black koji vinegar (Moromisu), since a wide variety of vinegar is commonly used as a beverage and frequently consumed in Japan. Additionally, we obtained a water-soluble BG (commercial name: Wellmune) to prepare a suitable beverage format. We selected the Moromisu vinegar, taken from the extraction of Awamori (typical Okinawa liquor) post-distillation slurry (Awamori-residue), 
which is produced during the course of Awamori production. Although the health benefits of the vinegar have not been scientifically proven, consumers still take the vinegar to maintain their healthy physical condition and well-being. In this study, a double-blind, randomized, placebo controlled, crossover trial was designed using a water-soluble BG formulated in the Moromisu. The vinegar contains amino acids, citric acid and other constituents; its general nutritional profile is described in Table 1. The vinegar by itself was the placebo and the Moromisu with added BG was the treatment sample. The nutritional profile of the Moromisu with added BG is also shown comparatively in Table 1. We employed the Profile of Mood State (POMS) survey to examine physical and psychological stress during a non-strenuous daily lifestyle for the first time. Previous studies have primarily tested subjects under strenuous exercise $[10,15]$.

Table 1. Nutritional composition of the sample vinegar $(100 \mathrm{ml})$

\begin{tabular}{|l|l|l|}
\hline & Vinegar (Placebo) & Vinegar + BG (300 mg) \\
\hline Energy (kcal) & 17 & 20 \\
\hline Protein (g) & 1.5 & 1.5 \\
\hline Lipid (g) & $<0.1$ & $<0.1$ \\
\hline Carbohydrates (g) & 2.7 & 3.2 \\
\hline Sodium (mg) & 10 & 11 \\
\hline Citric acid (g) & 0.3 & 0.3 \\
\hline Amino acid (mg) & & \\
\hline Ile & 59 & 59 \\
\hline Leu & 83 & 83 \\
\hline Lys & 72 & 72 \\
\hline Met & 20 & 20 \\
\hline Cys & 21 & 21 \\
\hline Phe & 57 & 57 \\
\hline Tyr & 73 & 73 \\
\hline Thr & 63 & 63 \\
\hline Trp & 9 & 9 \\
\hline Val & 81 & 81 \\
\hline His & 36 & 36 \\
\hline Arg & 122 & 122 \\
\hline Ala & 122 & 122 \\
\hline Asp & 122 & 122 \\
\hline Glu & 163 & 163 \\
\hline Gly & 83 & 83 \\
\hline Pro & 88 & 88 \\
\hline Ser & 71 & 71 \\
\hline & & \\
\hline & 53 & \\
\hline & 53 & \\
\hline
\end{tabular}

\section{METHODS}


Subjects: The study was conducted in accordance with the Declaration of Helsinki involving humans, and was also approved by the Institutional Review Board (IRB) of University of the Ryukyus (Approval No. 245). Subjects signed informed consent agreements after the study details were fully explained, and then screened on the inclusion/exclusion criteria as shown in Table 2. The subjects who met the criteria were selected for the study. Fourteen healthy men $(\mathrm{n}=10)$ and women $(\mathrm{n}=4)$ participated in the study. Characteristics of the subjects are described in Table 3.

Table 2. Inclusion and exclusion criteria used screening subjects for the study

\begin{tabular}{|l|l|}
\hline Inclusion criteria & - Subject must be $\geq 18$ years of age \\
& - Body mass index (BMI) must be $<25 \mathrm{~kg} / \mathrm{m}^{2}$ \\
& - Systolic blood pressure $<140 \mathrm{mmHg}$ and diastolic blood pressure $<90 \mathrm{mmHg}$ \\
- & Subjects who can follow the instructions during this study \\
\hline Exclusion criteria & - Smoker \\
& - Known allergy or sensitivity to any foods \\
& - Subject who uses any medicines or supplements \\
& - Family history of severe disease*
\end{tabular}

*Subject who have symptoms suggestive of possible cardiopulmonary or metabolic disease (American College of Sports Medicine Guidelines for Exercise Testing and Prescription $9^{\text {th }}$ Ed. Philadelphia (PA): Lippincott Williams \& Wilkins; 2014).

Preparation of research sample: Test samples for the study were prepared by Chuko Awamori Distillery Co., Ltd. (Okinawa, Japan). Each sample was poured into dark brown glass containers with aluminum-screwed caps, containing placebo vinegar (concentrated vinegar, water, indigestible dextrin, sucralose, sweetener and flavor) or vinegar with $300 \mathrm{mg}$ of a water-soluble BG supplied by Biothera, Inc. (Eagan, MN USA) as treatment sample. The nutritional profile of the placebo and treatment material is provided in Table 3. The content of BG in the treatment sample was confirmed by analysis, using a previously reported assay method [18]. All samples were provided to the research team using random code numbers. 
Table 3 The profile of the participated subjects in the study

\begin{tabular}{|l|l|}
\hline Gender ratio & Men: Women=10:4 \\
\hline Age & $22.8 \pm 0.6(21 \sim 30)$ \\
\hline Height $(\mathrm{cm})$ & $169.4 \pm 2.0$ \\
\hline Weight $(\mathrm{kg})$ & $61.5 \pm 2.5$ \\
\hline BMI $\left(\mathrm{kg} / \mathrm{m}^{2}\right)$ & $21.3 \pm 0.5$ \\
\hline SBP/DBP $(\mathrm{mmHg})$ & $108 \pm 3 / 64 \pm 2$ \\
\hline Hemoglobin concentration $(\mathrm{g} / \mathrm{dl})$ & $13.9 \pm 0.3$ \\
\hline
\end{tabular}

The values are mean \pm SEM.

Experimental design: A randomized, placebo-controlled, double-blind, crossover study with an 11-day washout period was performed between October 20 and December 22, 2014. Each subject took a 50-ml serving of the vinegar product, with or without $300 \mathrm{mg}$ of BG, for 10 days. Subjects were randomly assigned to placebo (vinegar) and treatment (vinegar and BG). In the crossover study, each subject ingested the sample beverage with or without the BG for 10 days. An 11-day washout period followed, before crossover to the alternate treatment or placebo for a subsequent 10-day period. The POMS survey was conducted on day 1 , day 10 , day 21 , and day 31 as described in Figure 1.

Profile of mood states: We employed the Profile of Mood States (POMS) survey [19, 20] to assess 6 primary psychological factors (tension, depression, anger, fatigue, vigor or confusion). This methodology is widely known and well-established [19]. The POMS questionnaire consists of 65 items, with each item scored on a $0-4$ scale ( $0=$ not at all, $4=$ extremely). The 65 adjective responses are classified into the aforementioned 6 factors, and data is collected, tabulated, scored and analyzed in accordance with the POMS methodology. 


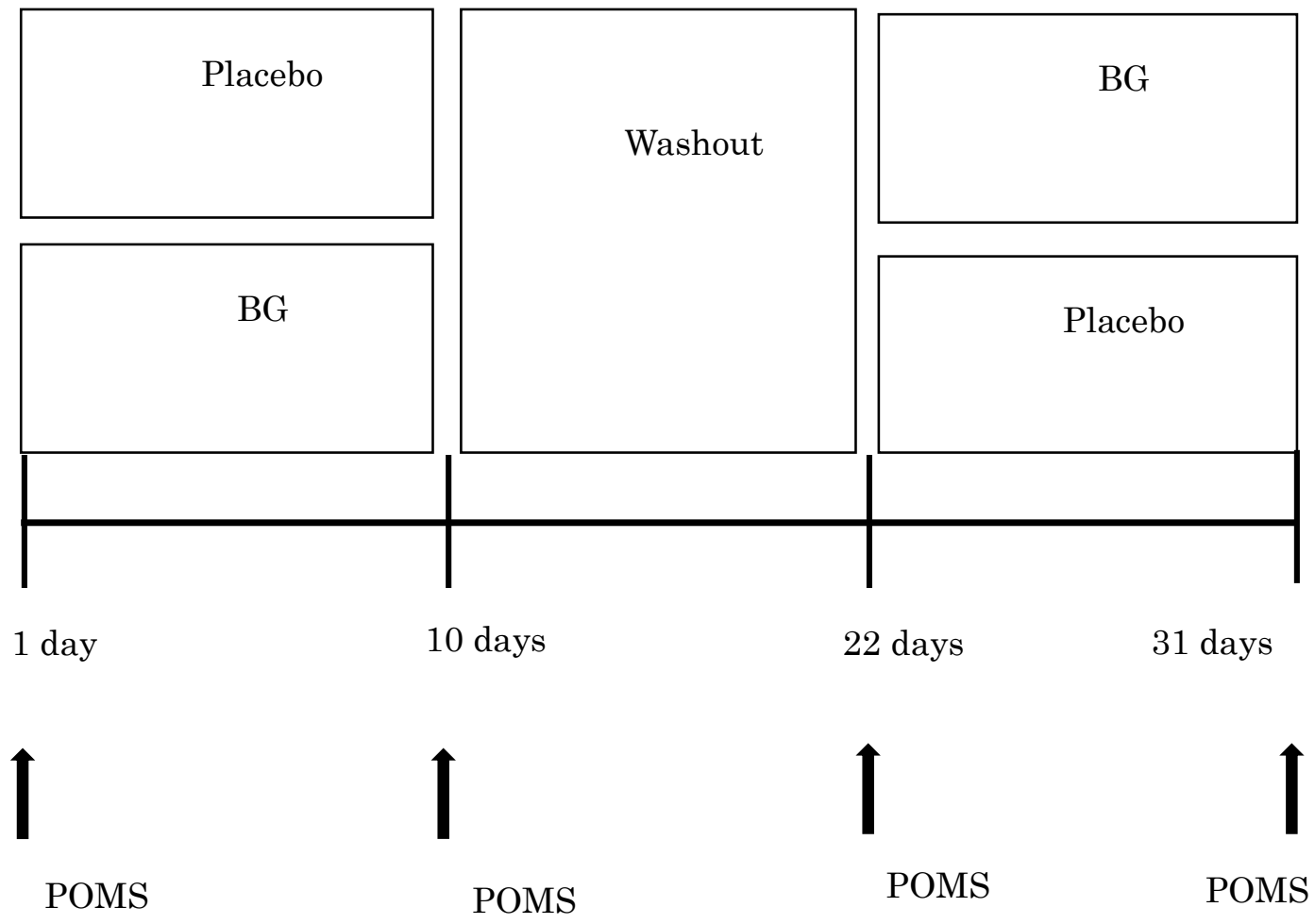

Figure 1. Flow of Experimental Procedure. All subjects received details of the protocol and procedure prior to the test. The trial dates were October 22 to December 20, 2014. The POMS survey (shown as POMS) was conducted at days 1, 10, 22 and 31.

Statistical Analysis: All questionnaires were collected by the research team. Data was identified by subject number, and was also checked for accuracy and completeness. The obtained data was analyzed using Welch's $t$-test, where differences were considered significant at $p<0.05$.

\section{RESULTS}

The statistical analyses reported the test statistic $p$-value. All data is expressed as mean $\pm \mathrm{SEM}$, unless otherwise specified. The study aimed to measure the factors in POMS, to evaluate the effects of BG on stress derived from non-strenuous daily lifestyle, including sedentary and slight to moderately exercise-oriented lifestyle in healthy subjects. No subjects dropped out of the study; every subject completed the test.

We noted that mood state responses for fatigue and confusion were significantly $(\mathrm{p}<0.05)$ reduced following 10 days of supplementation with BG (Figure 2) vs the start of the treatment period. 

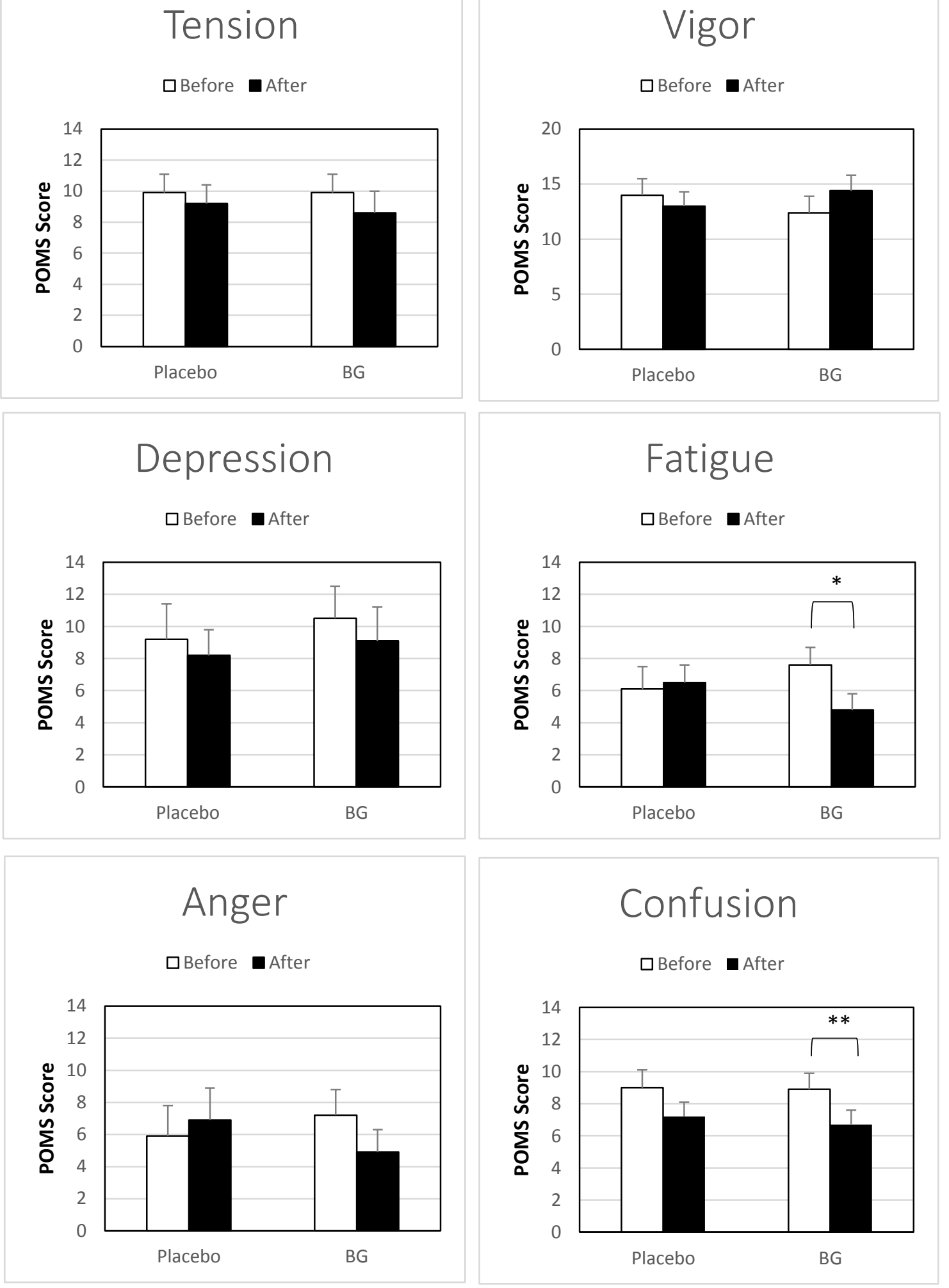

Figure 2. The results of POMS survey are shown for all subjects $(n=14)$. Statistically significant differences in scores were observed in the fatigue and vigor factors.

Each value was significantly different from those at the start of the treatment period: ${ }^{*} p<0.05,{ }^{* *} p<0.01$. 


\section{DISCUSSION}

The present study used a crossover design, where we ensured a sufficient washout period (11 days), while a previous study used a 7-day washout period [15]. A longer washout period allows subjects to experience further normalization of their daily lifestyle, since intake of the samples or any of the vinegar-based nutritional beverages is not a common practice for all subjects. In the present trial, $300 \mathrm{mg}$ of BG was added in consideration of the vinegar beverage formula used; in previous studies, $250 \mathrm{mg}$ and $500 \mathrm{mg}$ were found to be effective in improving POMS survey scores $[9,11-13]$ when taken in capsule form.

Overall, we observed significant reductions in the fatigue and confusion factors, in addition to strong statistical trends towards improved anger and vigor factors in the POMS methodology (anger; $p=0.054$, vigor; $p=0.056$ ). Although the number of subjects in the study was small and the supplement period (10 days) was short, we detected significant or trend differences in some of the POMS factors. Previous studies using POMS questionnaires for subjects taking BG as a supplement evaluated subjects who had undergone strenuous physical exercise, such as running for long distances $[5,16]$. Findings in this present study imply that even healthy subjects who have a non-strenuous daily lifestyle may still experience a certain extent of fatigue. Consequently, one may influence stress levels experienced daily by supplementing with BG in the diet, regardless of the form of ingestion. Therefore, a large-scale randomized control trial or epidemiologically designed study should be conducted in the future in order to support the findings of this study, including further evaluation of the immune system parameters and blood chemistry.

\section{CONCLUSIONS:}

The current study indicates a water-soluble BG from Saccharomyces cerevisiae in a vinegar beverage consumed by healthy subjects for 10 days may reduce indicators of stress and fatigue, based upon the POMS survey.

\section{LIST OF ABBREVIATIONS USED}

POMS: profile of mood states, URTI: upper respiratory tract infection, BG: beta 1,3/1,6 glucan.

Competing Interests: The authors have no financial interests or conflicts of interests 
Author's Contributions: All authors contributed to the study.

Acknowledgment: We thank Biothera Inc. (Eagan, MN USA) for supplying a water-soluble beta-glucan (Wellmune) for the study.

Funding: The study was supported by Chuko Awamori Distilllery Co., Ltd., Okinawa, Japan.

\section{REFERENCES:}

1. Cohen S, Doyle WJ, Skoner DP.: Psychological stress, cytokine production, and severity of upper respiratory illness. Psychosomatic Med. 1999; 61: 175-180.

2. Dhabhar FS.: Enhancing versus suppressive effects of stress on immune function: implications for immunoprotection and immunopathology. Neuroimmunomodulation 2009; 16: 300-317.

3. Carpenter KC, Breslin WL, Davidson T, et al.: Baker's yeast $\beta$-glucan supplementation increases monocytes and cytokines post-exercise: implications for infection risk? $\mathrm{Br} J$ Nutr. 2013; 109: 478-486.

4. Walsh NP, Gleeson M, Shephard RJ, et al:: Position statement. Part one: Immune function and exercise. Exerc Immunol Rev. 2011; 17: 6-63.

5. Cohen S, Janicki-Deverts D.: Who's Stressed? Distributions of Psychological Stress in the United States in Probability Samples from 1983, 2006, and 2009. J Appl Soc Psychol. 2012; 42: 1320-1334.

6. Nieman DC, Johanssen LM, Lee JW.: Infectious episodes in runners before and after the Los Angeles Marathon. J Sports Med Phys Fitness. 1990; 30: 316-328.

7. Peters EM, Bateman ED.: Ultramarathon running and upper respiratory tract infections. An epidemiological survey. S Afr Med J. 1983; 64: 582-584.

8. Peters EM, Goetzsche JM, Grobbelaar B.: Vitamin C supplementation reduces the incidence of postrace symptoms of upper-respiratory-tract infection in ultramarathon runners. Am J Clin Nutr. 1993; 57: 170-174.

9. Feldman S, Kalman D., Mayers A. et al.: Randomized phase II clinical trials of Wellmune WGP for immune support during cold and flu season. J Appl Res Clin Exp Ther. 2009; 9:30-42. 
10. Talbott S, Talbott J.: Effect of BETA 1,3/1, 6 GLUCAN on upper respiratory tract infection symptoms and mood state in marathon athletes. J Sports Sci Med. 2009; 8: 509-515.

11. Talbott S, Talbott J.: Beta 1,3/1,6 glucan decreases upper respiratory tract infection symptoms and improves psychological wellbeing in moderate to highly-stressed subjects. Agro Food Industry Hi-tech. 2010; 21: 21-24.

12. Fuller R, Yam T, Butt H, et al.: Influence of yeast-derived 1,3/1,6 glucopolysaccharide on circulating cytokines and chemokines and on upper respiratory tract infection symptoms in healthy adults. Nutrition. 2012; 28:665-669.

13. Talbott S, Talbott J.: Baker's Yeast Beta-Glucan Supplement Reduces Upper Respiratory Symptoms and Improves Mood State in Stressed Women. J Am Col Nutr. 2012; 31:1-6.

14. Talbott SM, Talbott JA, Talbott TL, et al.: $\beta$-Glucan supplementation, allergy symptoms and quality of life in self-described ragweed allergy sufferers. Food Sci Nutr. 2012; 10: 1-12.

15. McFarlin BK, Carpenter KC, Davidson T, et al.: Baker's Yeast Beta Glucan supplementation increase salivary IgA and decreases cold/flu symptomatic days after intense exercise. J Diet Suppl. 2013; 10: 171-183.

16. Li FX, Jin B, Liu W. et al.: Follow-up Formula Consumption in 3- to 4-Year-Olds and Respiratory Infections: An RCT. Pediatrics. 2014; 133: e1533-1540.

17. Scalabrin D, Ribeiro T, de Matto AP, et al.: Formula with docosahexaenoic acid, prebiotics, and beta-glucan supports respiratory and skin health in children. The FASEB Journal , 2014; 28: Supplement 382.6

18. Danielso ME, Dauth R, Elmasry NA.: Enzymatic method to measure $\beta-1,3-\beta-1,6$-glucan content in extracts and formulated products (GEM Assay). J Agric Food Chem. 2011; 58: $10305-10308$.

19. McNair D, Heuchert J, Shilony E.: Profile of mood states bibliography. 1964-2002. Multi-Health Xystems: North Tonowanda, NY (2003),

20. McNair DM, Lorr M, Droppleman LF (Eds.).: Manual for the profile of mood states, Education and Industrial Testing. Service, San Diego (1981). 